\title{
Incidência de dermatite associada à incontinência em pacientes de unidade de internação clínica
}

\author{
Incidence of incontinence-associated dermatitis among clinical inpatients
}

\author{
Incidencia de la dermatitis asociada a la incontinencia en pacientes de unidad de internación clínica
}

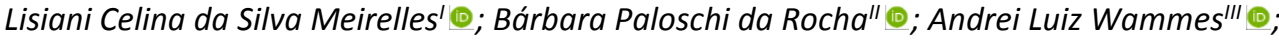 \\ Daiane Madruga dos Santos ${ }^{\prime V_{\odot}}$; Débora Monteiro da Silva ${ }^{{ }}$; Luccas Melo de Souza ${ }^{V I}{ }_{\odot}$

\begin{abstract}
'Hospital Moinhos de Vento, Porto Alegre, RS, Brasil; "Hospital Mãe de Deus, Porto Alegre, RS, Brasil; 'IHospital Bom Jesus, Porto Alegre, RS, Brasil; " Universidade Unisinos, Porto Alegre, RS, Brasil; 'Universidade Luterana do Brasil, Canoas, RS, Brasil; V'Universidade Federal de Ciências da Saúde de Porto Alegre, Porto Alegre, RS, Brasil
\end{abstract}

\begin{abstract}
RESUMO
Objetivo: identificar a incidência de Dermatite Associada à Incontinência (DAI) e os fatores de risco em pacientes de uma unidade de internação clínica. Método: estudo de coorte prospectivo com 197 pacientes de unidade de internação clínica. Utilizou-se estatística descritiva e analítica e calculou-se o Risco Relativo com intervalo de confiança de 95\%, considerando-se significativos quando $\mathrm{p}$ bicaudal <0,05. Estudo aprovado pelo Comitê de Ética em Pesquisa da Instituição. Resultados: a incidência de DAl foi de 20,3\% (40 pacientes). O risco relativo para DAl foi significativamente maior em idosos, em uso de alimentação enteral, fraldas, oxigenoterapia, antifúngico, corticoide, glicosídeo cardíaco, vasodilatador ou antidispéptico. Também foi maior nos pacientes com 15 ou mais dias de internação ou naqueles com doenças classificadas como Transtornos Mentais e Comportamentais ou como Causas Externas Traumáticas. Conclusão: foi alta a incidência de DAI na população pesquisada, estando associada a fatores de risco que envolvem, sobretudo, dependência de cuidado.

Descritores: Enfermagem; Dermatite; Incontinência Urinária; Incontinência Fecal.
\end{abstract}

\section{ABSTRACT}

Objective: to ascertain the incidence of Incontinence-Associated Dermatitis (IAD) and related risk factors among inpatients in a clinical unit. Method: in this prospective cohort study of 197 patients in an inpatient unit, descriptive and analytical statistics were used, and Relative Risk was calculated with a 95\% confidence interval; these were considered significant at two-tailed $p<$ 0.05. The research protocol was approved by the ethics commission. Results: incidence of IAD was $20.3 \%$ (40 patients). The relative risk IAD was significantly higher in the elderly, when using enteral feeding, diapers, oxygen therapy, antifungal, corticosteroids, cardiac glycoside, vasodilator or anti-dyspeptic. It was also higher in those with 15 or more days' hospitalization or with diseases classified as Mental and Behavioral Disorders or from External Traumatic Causes. Conclusion: the incidence of AID was high in the study population, and associated with risk factors that involved, above all, dependence on care.

Descriptors: Nursing; Dermatitis; Urinary Incontinence; Fecal Incontinence.

\section{RESUMEN}

Objetivo: determinar la incidencia de dermatitis asociada a incontinencia (DAI) y factores de riesgo relacionados entre pacientes hospitalizados en una unidad clínica. Método: en este estudio de cohorte prospectivo de 197 pacientes en una unidad de internación, se utilizó estadística descriptiva y analítica y se calculó el Riesgo Relativo con un intervalo de confianza del 95\%; estos se consideraron significativos en $\mathrm{p}<0,05$ de dos colas. El protocolo de investigación fue aprobado por la comisión de ética. Resultados: la incidencia de DAl fue del 20,3\% (40 pacientes). El riesgo relativo de DAI fue significativamente mayor en los ancianos, al utilizar alimentación enteral, pañales, oxigenoterapia, antifúngicos, corticosteroides, glucósidos cardíacos, vasodilatadores o antidispépticos. También fue mayor en aquellos con 15 o más días de hospitalización o con enfermedades clasificadas como trastornos mentales y del comportamiento o de causas traumáticas externas. Conclusión: la incidencia de DAI fue alta en la población de estudio, y se asoció a factores de riesgo que involucraron, sobre todo, dependencia del cuidado. Descriptores: Enfermería; Dermatitis; Incontinencia Urinaria; Incontinencia Fecal.

\section{INTRODUÇÃO}

A Dermatite Associada à Incontinência (DAI) é um tipo de dermatite de contato que ocorre pela exposição prolongada da pele a urina e/ou fezes, afetando o períneo ou áreas adjacentes ${ }^{1}$. Abrange um conjunto de alterações que provocam inflamação da superfície da pele, caracterizando-se, especialmente, por eritema brilhante, aparência macerada, com ou sem erosão da epiderme, acompanhada de desconforto ou dor semelhante à queimadura. Pode manifestar-se, ainda, por edema, vesículas, crostas ou descamação da pele, caracterizando o eczema ${ }^{2,3}$.

O desenvolvimento de DAl ocorre a partir de combinação de substâncias químicas irritantes (ureia, amônia e enzimas digestivas) que entram em contato com a pele, associadas à umidade, fricção, colonização por microrganismos, duração e frequência da exposição. A combinação desses fatores, originados da incontinência fecal e/ou urinária, pode alterar o $\mathrm{pH}$ da pele e predispor o paciente à $\mathrm{DAl} \mathrm{I}^{1,4}$.

Autora correspondente: Luccas Melo de Souza. E-mail: luccasms@gmail.com

Editora responsável: Flavia Giron Camerini 
Nesse sentido, o cuidado de enfermagem para com pacientes com incontinência é um desafio, pois sua grande incidência implica em agravos na pele, o que interfere nos indicadores de qualidade assistencial e de segurança do paciente ${ }^{5}$.

Em pacientes internados, a incontinência é um problema cotidiano. Estudo realizado em 36 estados dos Estados Unidos da América (EUA) identificou algum tipo de incontinência em 2492 (46,6\%) sujeitos da amostra, de um total de 5342 pacientes em cuidados agudos. A taxa geral de prevalência de DAl foi de $21,3 \%(1140 / 5342)$ e a prevalência entre aqueles com incontinência foi de 45,7\% (1140/2492) .

No Brasil, dados sobre DAI ainda são incipientes, sobretudo no sistema público de saúde e em ambientes de cuidados não agudos, como em unidades de internação clínica, havendo, carência de dados nacionais ${ }^{7}$. Portanto, o estudo justifica-se pela necessidade de dados científicos nacionais sobre o tema e pela elevada frequência de pacientes incontinentes em uso de fraldas, sobretudo por conta do envelhecimento da população e do aumento das doenças crônicas e das internações hospitalares repetidas e prolongadas ${ }^{5,8}$.

Neste cenário de considerações, este estudo tem como objetivo: identificar a incidência de DAl e os fatores de risco em pacientes de uma unidade de internação clínica.

\section{MÉTOdO}

Trata-se de um estudo de coorte prospectivo, desenvolvido em uma unidade de internação clínica de um hospital da Região Metropolitana de Porto Alegre, Rio Grande do Sul, Brasil.

A unidade campo de estudo comporta 62 leitos, sendo seis psiquiátricos, sete pediátricos e 49 clínicos. Dispõe, na equipe de enfermagem, de dois enfermeiros em cada turno (manhã/tarde), um em cada noite (noite A e B) e 13 técnicos de enfermagem por turno. Todos os pacientes dessa unidade são atendidos via convênio público do Sistema Único de Saúde (SUS). No ano de 2015, a referida unidade atendeu 2.002 pacientes adultos/idosos na área clínica, conforme informações obtidas junto ao setor de recursos humanos da instituição.

A população desta pesquisa compreendeu todos os pacientes admitidos na unidade de internação clínica do campo de estudo durante o período de coleta de dados, iniciado em 1 de outubro de 2016. Os critérios de inclusão adotados foram: possuir idade igual ou superior a 18 anos; internar na unidade a partir de 1 de outubro de 2016; estar internado em um dos 49 leitos clínicos da unidade em um dos dias da coleta de dados (segundas, quartas e sextasfeiras). Foram incluídos pacientes com ou sem uso de fraldas, bem como os continentes e incontinentes, a fim de se evitar viés de seleção, uma vez que se pretendia identificar a incidência de DAI na unidade de internação. A seleção dos novos sujeitos, para ingresso no estudo, considerava aqueles com menor tempo de internação na unidade campo de estudo no dia, além dos critérios de inclusão iniciais.

Os critérios de exclusão foram: possuir DAI na admissão na unidade, ter nova internação na unidade após participar da coleta de dados na internação anterior ou, ainda, ser transferido de outra instituição.

Considerou-se, para o cálculo amostral, o total de 2.002 internações de pacientes clínicos na unidade campo de estudo (no ano de 2015 ), a prevalência de DAI de $15 \%{ }^{9}$, erro amostral de $5 \%$ e o intervalo de confiança de $95 \%$.

Com base nos parâmetros anteriormente citados, o tamanho amostral calculado foi de 179 pacientes a serem acompanhados. Porém, por conta de possíveis perdas e recusas, houve um acréscimo de $10 \%$, de forma que a amostra final do estudo compreendeu 197 pacientes.

A coleta dos dados foi realizada por um enfermeiro com titulação de doutor e por acadêmicos de enfermagem, os quais foram submetidos à capacitação que incluiu abordagem teórico-prática sobre a avaliação sistemática de DAI e da pele em geral. A DAI foi identificada por exame físico e, quando presente ou em suspeita, o pesquisador principal era contatado para validar o achado.

Os pesquisadores avaliaram todos os 197 pacientes do estudo (internados na unidade, conforme critérios de inclusão/exclusão) nas segundas, quartas e sextas-feiras (inclusive quando em feriados), durante os meses de outubro a dezembro de 2016. A coleta de dados foi realizada somente com os pacientes que concordaram em participar do estudo ou com a autorização do seu responsável, por meio de assinatura do Termo de Consentimento Livre e Esclarecido.

Preconizou-se, em função de se obter melhor qualidade no registro das informações, o acompanhamento diário sistemático de 25 sujeitos, ou seja: a cada dia, avaliavam-se 25 pacientes (entre novos e os já incluídos no estudo). Portanto, iniciou-se a coleta de dados com sorteio aleatório de 25 pacientes dos 49 possíveis. À medida que cada sujeito recebia alta, incluíam-se novos pacientes para se manter o acompanhamento diário de 25 indivíduos. Cada paciente foi acompanhado do início ao término da sua internação na unidade, independentemente do desfecho (alta hospitalar, transferência ou óbito), até se alcançar o total de 197 pacientes. 
Inicialmente, consultava-se o prontuário dos pacientes para, posteriormente, realizarem-se o exame físico e o registro dos dados no instrumento de coleta de dados. Utilizou-se Termo de Compromisso para Utilização dos Dados de prontuário, a fim de se manter o anonimato dos pacientes e uso dos dados somente para fins científicos.

O instrumento para coleta de dados abordou dados sociodemográficos, clínicos e específicos sobre DAI. As seguintes variáveis foram coletadas a beira do leito, por anamnese e exame físico, em cada dia de coleta de dados: presença de DAl, cor da pele autorreferida, via de alimentação, oxigenação, tipo de incontinência, uso de fraldas, peso, altura, autocuidado com eliminações e fezes líquidas. As demais foram coletadas no prontuário do paciente (idade, sexo, uso de medicamentos na internação e diagnósticos médicos no momento da internação).

A avaliação dos sinais e sintomas de DAl ocorreu por exame físico, uma vez ao dia (nas segundas, quartas e sextasfeiras), quando a equipe assistencial realizava a higiene perineal, troca de fraldas ou banho do paciente.

Os diagnósticos médicos foram extraídos do prontuário do paciente (no dia em que cada paciente foi incluído no estudo) e agrupados conforme a Classificação Internacional das Doenças, versão 10 (CID-10) ${ }^{10}$. O uso de medicamentos durante a internação também foi extraído do prontuário do paciente (em cada dia de coleta) na prescrição médica do dia.

As informações de peso e altura foram coletadas conforme relato do paciente ou, como segunda opção, do prontuário, conforme registro do serviço de nutrição da instituição. Não se obteve tal informação de todos os pacientes da amostra. Utilizou-se o IMC da primeira coleta de dados para cada paciente, ou seja, aquele quando da internação do paciente na unidade.

Para a classificação do Índice de Massa Corporal (IMC), foi utilizada a definição da Organização Mundial de Saúde ${ }^{11}$ : magreza (menor que 18,5 kg/m²), normal (entre 18,5 a 24,9 Kg/m²), sobrepeso ( 25 a 29,9 Kg/m²), obesidade (entre 30 e 39,9 $\mathrm{Kg} / \mathrm{m}^{2}$ ) e obesidade grave (a partir de $40 \mathrm{Kg} / \mathrm{m}^{2}$ ). Após, a variável foi dicotomizada em normal e alterado (demais categorias).

A análise dos dados ocorreu pelo programa Statistical Package for Social Science for Windows, utilizando-se estatística descritiva (frequência, percentual, média, desvio padrão e incidência) e analítica (Risco Relativo com intervalo de confiança de 95\%), considerando estatisticamente significativos os valores com $\mathrm{p}$ bicaudal $<0,05$.

A pesquisa foi aprovada pelo Comitê de Ética em Pesquisa da instituição proponente (Parecer $\left.n^{\circ} 1.755 .453 / 2016\right)$, em conformidade com a Resolução n. 466/2012, do Conselho Nacional de Saúde. A participação no estudo envolveu riscos mínimos ao paciente, visto que não houve intervenções, somente entrevista e exame físico, sendo esses percebidos como desconforto pelo tempo e pela manipulação dos sujeitos.

\section{RESULTADOS}

A caracterização da amostra de 197 pacientes é apresentada na Tabela 1.

TABELA 1: Incidência de DAl e Risco Relativo conforme variáveis demográficas e de saúde. Região Metropolitana de Porto Alegre, RS, Brasil, 2016.

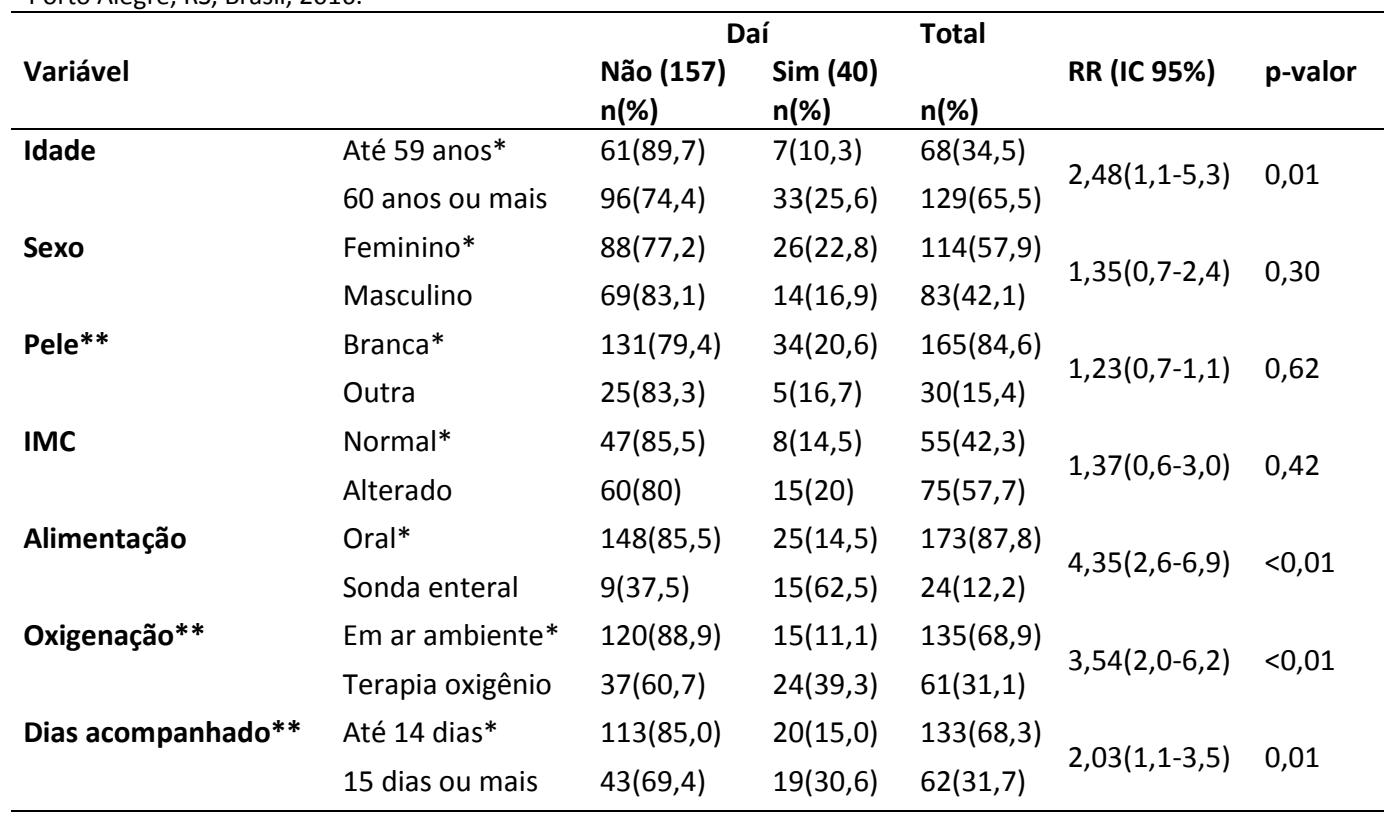

${ }^{*}$ Categoria de referência; ${ }^{* *} \mathrm{n}<197 ;$ RR: Risco Relativo. 
A amostra caracteriza-se por ser predominantemente feminina, com média de idade de $64,2 \pm 17,6$ anos, de pele branca, com IMC alterado, recebendo alimentação via oral e ventilando em ar ambiente. A média do IMC foi de 25,6士5,6. A incidência de DAl foi de 20,3\% (40 pacientes).

Foi estatisticamente maior o risco para DAI nos idosos (2,48 vezes), nos pacientes em uso de alimentação por sonda enteral (4,35 vezes), em terapia com oxigênio (3,54 vezes) e com 15 ou mais dias de internação (2,03 vezes).

Os resultados da análise de risco para DAl são apresentados na Tabela 2.

TABELA 2: Incidência de DAl e Risco Relativo conforme variáveis relacionadas às eliminações urinárias e fecais. Região Metropolitana de Porto Alegre, RS, Brasil, 2016

\begin{tabular}{|c|c|c|c|c|c|}
\hline \multirow[b]{2}{*}{ Variável } & \multicolumn{2}{|c|}{ Daí } & \multirow{2}{*}{$\begin{array}{l}\text { Total } \\
\mathrm{n}(\%)\end{array}$} & \multirow[b]{2}{*}{ RR (IC 95\%) } & \multirow[b]{2}{*}{ p-valor } \\
\hline & $\begin{array}{c}\text { Não (157) } \\
\text { n(\%) }\end{array}$ & $\begin{array}{c}\operatorname{Sim}(40) \\
n(\%)\end{array}$ & & & \\
\hline \multicolumn{6}{|c|}{ Incontinência urinária } \\
\hline Não* & $132(98,5)$ & $2(1,5)$ & $134(68,1)$ & \multirow{2}{*}{$40,41(10,0-162,2)$} & \multirow{2}{*}{0,01} \\
\hline Sim & $25(39,7)$ & $38(60,3)$ & $63(31,9)$ & & \\
\hline \multicolumn{6}{|l|}{ Incontinência fecal } \\
\hline Não* & $124(96,9)$ & $4(3,1)$ & $128(65,0)$ & \multirow{2}{*}{$16,69(6,2-44,9)$} & \multirow{2}{*}{$<0,01$} \\
\hline Sim & $33(47,8)$ & $36(52,2)$ & $69(35,0)$ & & \\
\hline \multicolumn{6}{|l|}{ Dupla incontinência } \\
\hline Não* & $134(95,7)$ & $6(4,3)$ & $140(71,1)$ & \multirow[b]{2}{*}{$13,91(6,1-31,3)$} & \multirow[b]{2}{*}{$<0,01$} \\
\hline Sim & $23(40,4)$ & $34(59,6)$ & $57(28,9)$ & & \\
\hline \multicolumn{6}{|l|}{ Uso de fraldas } \\
\hline Não* & $127(97,7)$ & $3(2,3)$ & $130(67,5)$ & \multirow{2}{*}{$23,9(7,6-74,5)$} & \multirow{2}{*}{$<0,01$} \\
\hline $\operatorname{Sim}$ & $30(44,8)$ & $37(55,2)$ & $67(32,5)$ & & \\
\hline \multicolumn{6}{|l|}{ Uso de duas fraldas } \\
\hline Não* & $21(50,0)$ & $21(50,0)$ & $42(62,7)$ & \multirow{2}{*}{$1,28(0,8-1,9)$} & \multirow{2}{*}{0,26} \\
\hline Sim & $9(36,0)$ & $16(64,0)$ & $25(37,3)$ & & \\
\hline \multicolumn{6}{|l|}{ Autocuidado urina } \\
\hline Independente* & $112(99,1)$ & $1(0,9)$ & $113(57,4)$ & \multirow{2}{*}{$62,46(7,3-374,2)$} & \multirow{2}{*}{$<0,01$} \\
\hline Com dependência & $45(53,6)$ & $39(46,4)$ & $84(42,6)$ & & \\
\hline \multicolumn{6}{|l|}{ Autocuidado fezes } \\
\hline Independente* & $122(97,6)$ & $3(2,4)$ & $125(63,5)$ & \multirow{2}{*}{$21,41(6,8-66,9)$} & \multirow{2}{*}{$<0,01$} \\
\hline Com dependência & $35(48,6)$ & $37(51,4)$ & $72(36,5)$ & & \\
\hline \multicolumn{6}{|l|}{ Fezes Líquidas } \\
\hline Não* & $133(82,6)$ & $28(17,4)$ & $161(81,7)$ & \multirow{2}{*}{$1,91(1,1-3,3)$} & \multirow{2}{*}{0,03} \\
\hline Sim & $24(66,7)$ & $12(33,3)$ & $36(18,3)$ & & \\
\hline
\end{tabular}

${ }^{*}$ Categoria de referência; RR: Risco Relativo.

O risco para DAI foi estatisticamente maior nos pacientes com incontinência urinária (40,4 vezes), fecal (16,6 vezes) ou dupla (13,9 vezes). Foi também maior naqueles em uso de fraldas (23,9 vezes), dependentes para autocuidado com urina (62,4 vezes maior) e fezes (21,4 vezes) e naqueles que apresentaram fezes líquidas (1,91 vezes).

Já os resultados relativos à incidência de DAl e Risco Relativo conforme doenças diagnosticadas e uso de medicamentos é apresentado na Tabela 3. 
TABELA 3: Incidência de DAI e Risco Relativo conforme doenças diagnosticadas e uso de medicamentos. Região Metropolitana de Porto Alegre, RS, Brasil, 2016.

\begin{tabular}{|c|c|c|c|c|c|}
\hline \multirow{3}{*}{ Variável } & \multicolumn{2}{|c|}{ Daí } & \multirow[t]{2}{*}{ Total } & \multirow{3}{*}{ RR (IC 95\%) } & \multirow{3}{*}{ p-valor } \\
\hline & Não (157) & $\operatorname{Sim}(40)$ & & & \\
\hline & $\mathrm{n}(\%)$ & $n(\%)$ & $n(\%)$ & & \\
\hline \multicolumn{6}{|c|}{ Transtornos Mentais e Comportamentais } \\
\hline Não* & $156(80,4)$ & $38(19,6)$ & $194(98,4)$ & \multirow{2}{*}{$3,40(1,4-7,9)$} & \multirow{2}{*}{0,04} \\
\hline Sim & $1(33,3)$ & $2(66,7)$ & $3(1,6)$ & & \\
\hline \multicolumn{6}{|c|}{ Causas Externas Traumáticas } \\
\hline Não* & $156(80,8)$ & $37(19,2)$ & $193(97,9)$ & \multirow{2}{*}{$3,91(2,0-7,3)$} & \multirow{2}{*}{$<0,01$} \\
\hline Sim & $1(25,0)$ & $3(75,0)$ & $4(2,1)$ & & \\
\hline \multicolumn{6}{|c|}{ Corticoide } \\
\hline Não* & $137(83,0)$ & $28(17,0)$ & $165(83,7)$ & \multirow{2}{*}{$2,21(1,2-3,8)$} & \multirow{2}{*}{$<0,01$} \\
\hline Sim & $20(62,5)$ & $12(37,5)$ & $32(16,3)$ & & \\
\hline \multicolumn{6}{|c|}{ Vasodilatador } \\
\hline Não* & $148(81,8)$ & $33(18,2)$ & $181(91,8)$ & \multirow{2}{*}{$2,40(1,2-4,5)$} & \multirow{2}{*}{0,01} \\
\hline Sim & $9(56,2)$ & $7(43,8)$ & $16(8,2)$ & & \\
\hline \multicolumn{6}{|c|}{ Glicosídeo cardíaco } \\
\hline Não* & $156(80,4)$ & $38(19,6)$ & $194(98,4)$ & \multirow{2}{*}{$3,40(1,4-7,9)$} & \multirow{2}{*}{0,04} \\
\hline Sim & $1(33,3)$ & $2(66,7)$ & $3(1,6)$ & & \\
\hline \multicolumn{6}{|c|}{ Antifúngico } \\
\hline Não* & $156(80,4)$ & $38(19,6)$ & $194(98,4)$ & \multirow{2}{*}{$3,40(1,4-7,9)$} & \multirow{2}{*}{0,04} \\
\hline Sim & $1(33,3)$ & $2(66,7)$ & $3(1,6)$ & & \\
\hline \multicolumn{6}{|c|}{ Antidispéptico } \\
\hline Não* & $154(81,1)$ & $36(18,9)$ & $190(96,4)$ & \multirow{2}{*}{$3,01(1,4-6,1)$} & \multirow{2}{*}{0,01} \\
\hline Sim & $3(42,9)$ & $4(57,1)$ & $7(3,6)$ & & \\
\hline
\end{tabular}

*Categoria de referência; RR: Risco Relativo.

Quantos às doenças, conforme CID-10, foi maior o risco para DAI nos pacientes cujas doenças de base foram diagnosticadas como Transtornos Mentais e Comportamentais (3,4 vezes) ou como Causas Externas Traumáticas (3,91 vezes). No que se refere aos medicamentos, foi maior o Risco Relativo naqueles em uso de corticoides $(2,21$ vezes), vasodilatador (2,4 vezes), glicosídeo cardíaco (3,4 vezes), antifúngico (3,4 vezes) e antidispéptico (3,01 vezes).

\section{DISCUSSÃo}

A incidência de DAl foi de 20,3\% entre os 197 pacientes, sendo que 63 (31,9\%), 69 (35\%) e 57 (28,9\%) tinham incontinência urinária, fecal ou dupla, respectivamente, o que demonstra uma problemática preocupante: um em cada 5 pacientes do estudo desenvolveu DAI. Estudos sobre incidência de DAI são escassos, pois a maior parte deles é de delineamento transversal, limitando a comparação dos achados deste estudo. Consensos e revisões indicam prevalência de DAl variando de 5,6\% a 50\% e incidência entre 3,4\% e 25\%, salientando que essas variações ocorrem, especialmente, pela dificuldade de se reconhecer a DAl como uma condição e também em diferenciá-la de outras lesões de pele, como as Lesões por Pressão ${ }^{1,12}$.

Na China, estudo longitudinal com 109 pacientes em cuidados intensivos identificou que a incidência de DAI foi de 23,9\%. Na análise univariada, foi maior a incidência em pacientes com risco nutricional, Lesão por Pressão, baixo nível de albumina, alguma infecção, incontinência fecal, maior frequência de incontinência fecal, tipo de fezes (líquidas) e dupla incontinência. $\mathrm{Na}$ análise multivariada, quanto maior o valor de albumina sérica (desde que dentro da faixa de normalidade), menor foi o risco para DAl; quanto menor o risco para LP (conforme escala de Braden), menor o risco para DAI. Ainda, dupla incontinência foi fator de risco independente para DAl ${ }^{13}$. Outros autores também reforçam a existência de maior risco de se desenvolver DAl em incontinentes fecais ou dupla, em comparação aos com somente incontinência urinária ${ }^{6,14}$.

Em contrapartida, este estudo identificou maior risco para DAl em pacientes com incontinência urinária (RR 40,41) em detrimento daqueles com incontinência fecal (RR 16,69) ou dupla (RR 13,91). Uma hipótese para explicar tais dados pode ser o fato de que pacientes com incontinência fecal (ou dupla) receberiam maior atenção para a troca de fraldas em comparação àqueles somente com incontinência urinária, ou seja: pode haver maior agilidade na troca de fraldas, na higiene e nos cuidados com a pele em pacientes com incontinência fecal - em virtude do maior desconforto e odor que causam - e, consequentemente, menos exposição. Essa situação tem mais chance de acontecer, especialmente, em unidades de internação do que em unidades de cuidado intensivo. Tal situação pode caracterizar efeito de confusão nos dados, pois não foi possível ter o controle das frequências de higienização na unidade e do tempo de exposição conforme tipo de eliminações, já que não foi o escopo do estudo realizar alguma intervenção na assistência. 
Salienta-se, também, a possibilidade de efeito modificador, comum em estudos longitudinais. Como não houve, nesse estudo, controle das variáveis antes e depois do desfecho, os resultados não permitem relação causa-efeito nas variáveis analisadas, apenas possibilitam inferir associação entre elas ${ }^{15}$.

O tempo de exposição à urina e a fezes é fator relacionado com incidência e prevalência de DAl devido à maior umidade e ao tempo de contato das substâncias químicas irritantes (ureia, amônia e enzimas digestivas) com a pele ${ }^{1}$. 0 estudo aponta que houve maior risco para DAI em indivíduos com fezes líquidas e com maior dependência para cuidados como auxílio para a troca de fraldas, complementação de oxigênio ou de nutrição enteral, e naqueles com maior tempo de internação hospitalar. Esses achados vão ao encontro de outros estudos que identificaram maior ocorrência de DAl em idosos, com fezes líquidas, com baixa saturação de oxigênio, com risco nutricional e com imobilidade, entre outras características $^{6,13,16}$. Em contrapartida, estudo brasileiro identificou que necessidade de suporte nutricional foi fator de proteção para DAl em pacientes críticos 5 .

Sobre o maior risco de DAI em pacientes com incontinência urinária, destaca-se que o uso de dispositivos como cateteres vesicais ou códons urinários (em homens) pode estabelecer melhores práticas para a prevenção e, sobretudo, tratamento de DAl, mesmo que as atuais diretrizes restrinjam o uso de dispositivos invasivos com vistas à redução de infecção do trato urinário ${ }^{17}$.

Ainda, estudo realizado com 5342 pacientes adultos internados em uma unidade de cuidado intensivo de 36 estados de todas as regiões dos Estados Unidos da América apontou prevalência de DAl de 21,3\%, sendo que 46,6\% dos pacientes eram incontinentes. Entre os incontinentes, a prevalência de DAl foi de 45,7\%, maior em pacientes com incontinência fecal $(44,7 \%)$ ou dupla $(49,2 \%)$ do que em pacientes somente com incontinência urinária $(29,7 \%)^{6}$.

Na Austrália, 245 (65,1\%) de 376 pacientes internados em cuidados intensivos tinham alguma incontinência, sendo que a DAl prevaleceu em $10 \%$ do total de pacientes e em $42 \%$ daqueles com incontinência. A taxa de prevalência de incontinência foi de $24 \%$. Fezes líquidas e pastosas foram associadas à DAI $(p=0,027)$, e a indicação clínica de infecção fúngica ocorreu em $32 \%$ dos pacientes com DAl ${ }^{18}$. No presente estudo, foi maior a incidência em pacientes em uso de antifúngicos (RR 3,40). No estudo norte-americano com pacientes em cuidado intensivo, 14.8\% (169/1140) dos que possuíam DAl também tinham erupção fúngica ${ }^{6}$.

O maior risco para infecções, como as fúngicas, é complexo e multifatorial. O pH mais alcalino (modificado pelo uso de sabonetes com pH maior que o da pele) aumenta o risco de colonização por microrganismos e, portanto, de infecções cutâneas, também agravado pelo atrito que ocorre pelo uso de compressas na higiene perineal. Junto a isso, o uso de fraldas com pouca capacidade de absorção e/ou pouco arejamento favorece o desenvolvimento de DAI pelo aumento da umidade, situação propícia para o desenvolvimento de fungos e bactérias, que pioram a característica da lesão e dificultam o tratamento ${ }^{4,5,19}$.

Com isso, é premente a necessidade de manter e melhorar a integridade da pele dos pacientes dependentes, especialmente aqueles com algum tipo de incontinência e em uso de fraldas. Além disso, é preciso incluir a avaliação da DAI na práxis do enfermeiro, em virtude do aumento progressivo de doenças crônicas e debilitantes que levam à dependência, à incontinência e à fragilidade da pele ${ }^{1,13}$.

Para isso, são necessárias a elaboração e a validação de protocolos de prevenção, sobretudo nas etapas de limpeza suave, hidratação e proteção com produtos de barreira. É notória a evolução no conhecimento a respeito da fisiopatologia e da epidemiologia da DAl, assim como há simultânea necessidade de maior evidência sobre quais produtos são os mais adequados para proteção e tratamento. Alguns demonstram boa efetividade para o tratamento e prevenção de DAl, como lenços umedecidos 3 em 1 (limpeza, hidratação e proteção) a base de dimeticona 3\%, protetor cutâneo em spray de acrilato, cremes de barreira e dispositivos de contenção fecal ${ }^{4,20-23}$.

Ainda, o achado de maior risco para DAl em pacientes com doenças diagnósticas como Transtornos Mentais e Comportamentais (RR 3,40) ou Causas Externas Traumáticas (RR 3,91) assemelha-se ao do estudo de coorte brasileiro com pacientes de duas unidades de tratamento intensivo, no qual pacientes com admissão por trauma apresentaram maior risco para $\mathrm{DAl}^{5}$. Nesse estudo, pacientes com diagnóstico médico de trauma apresentaram risco 16,11 vezes maior para desenvolver DAI. Acredita-se que tal associação pode estar relacionada à maior dificuldade na higiene perineal de pacientes com tais transtornos por conta do nível de consciência baixo (não percebem/controlam as eliminações) ou trauma (que dificulta a mobilização), o que pode influenciar no tempo de exposição e na qualidade das higienizações nesses casos.

Sobre o maior risco de DAl em pacientes que utilizavam corticoide, vasodilatadores, glicosídeo cardíaco e antidispéptico (além de antifúngicos), estudos específicos sobre DAl e medicamentos precisam ser realizados a fim de se compreender se há relação entre o uso específico de certos medicamentos ou se a polifarmácia (e, consequentemente, as condições de saúde do paciente) é aquilo que influencia a ocorrência de DAl. 


\section{Limitações do estudo}

As limitações do estudo incluem a amostra, que envolve pacientes atendidos pelo sistema público de saúde do Brasil de uma unidade de internação clínica de um hospital de médio porte que não ofertava treinamento específico para abordagem da DAl aos seus colaboradores; algumas variáveis de pesquisa coletadas somente na primeira avaliação (IMC e doenças, por exemplo) ou consideradas no contexto geral da internação, sem análise diária com a incidência de DAI (uso de medicamentos, por exemplo); a possibilidade de falta de precisão nas variáveis peso e altura, à medida que foi autorrelatada ou coletada do prontuário; o fato de a coleta de dados ter ocorrido três vezes na semana, o que pode gerar subavaliação da incidência de DAl; a possibilidade de vieses de aferição (à medida em que os pesquisadores não podiam ser cegados), de confundimento ou de modificação, visto que algumas variáveis não puderam ser coletadas (número de troca de fraldas/dia, qualidade das higienizações, tipo de fraldas) por não haver padronização na unidade.

\section{CONCLUSÃO}

A incidência de DAI pode ser considerada alta ( $1 / 5$ da amostra investigada) se comparada a resultados da literatura e por não se tratar de uma amostra de pacientes em cuidados críticos. O risco de DAl foi maior em pacientes idosos, com alimentação por sonda enteral, com mais dias de hospitalização, em uso de fraldas, dependentes para cuidados com eliminações, com fezes líquidas, que utilizaram alguns tipos de medicamentos (corticoide, vasodilatador, glicosídeo cardíaco, antifúngico ou antidispéptico) e com internação por doenças categorizadas como Transtornos Mentais e Comportamentais ou como Causas Externas Traumáticas. Entre os tipos de incontinência, foi maior o risco em incontinentes urinários.

Os resultados deste estudo identificam a DAI no contexto de um hospital brasileiro, trazendo evidências que podem ser utilizadas na prática assistencial para reconhecer pacientes com maior risco para DAI, melhorando a qualidade assistencial. Torna-se essencial a implementação de intervenções de enfermagem eficazes e viáveis para o cuidado com a pele, visto que o enfermeiro tem um importante papel nos cuidados de higiene e conforto. Apesar da considerável incidência, o assunto ainda é pouco pesquisado, sendo necessárias pesquisas com outras abordagens e em populações distintas.

\section{REFERÊNCIAS}

1. Beeckman D. A decade of research on Incontinence-Associated Dermatitis (IAD): evidence, knowledge gaps and next steps. J. Tissue Viability [Internet]. 2017 [cited 2020 May 14]; 26(1):47-56. DOI: https://doi.org/10.1016/j.jtv.2016.02.004.

2. Tavares AP, Ramos AF, Vieira EV, Mendonça S, Fonsenca $C$, Lopes $M$, et al. Nursing Intervention results in the prevention and healing of dermatitis associat. RIASE [Internet]. 2016 [cited 2020 Feb 16]; 2(3):709-22. Available from: http://www.revistas.uevora.pt/index.php/saude_envelhecimento/article/view/151.

3. Voegeli D. Incontinence-associated dermatitis: new insinghts into an old problem. Br J. Nurs. [Internet]. 2016 [cited 2020 May 16]; 25(5):256-2. DOI: https://doi.org/10.12968/bjon.2016.25.5.256.

4. Beele H, Smet S, Van Damme N, Beeckman D. Incontinence-associated dermatitis: pathogenesis, contributing factors, prevention and management options. Drugs Aging [Internet] 2018 [cited 2020 May 26]; 35(1):1-10. DOI: https://doi.org/10.1007/s40266-017-0507-1.

5. Chianca TMC, Gonçales PC, Salgado PO, Machado BO, Amorim GL, Alcoforado CLGC. Incontinence-associated dermatitis: a cohort study in critically ill patients. Rev. Gaúcha Enferm. [Internet]. 2016 [cited 2020 Feb 16]; 37(esp):e68075. DOI: http://dx.doi.org/10.1590/1983-1447.2016.esp.68075.

6. Gray M, Giuliano KK. Incontinence-associated dermatitis, characteristics and relationship to pressure injury. J. Wound Ostomy Continence Nurs. [Internet]. 2018 [cited 2020 Feb 13]; 45(1):63-7. Available from: https://www.ncbi.nlm.nih.gov/pubmed/29300291.

7. Nascimento DC, Cunha CV, Penna LHG, Souza NVDO, Marques GS. Dermatite associada à incontinência na população idosa: uma revisão integrativa. Rev. HUPE [Internet]. 2016 [cited 2020 Feb 16]; 15(1):37-2. DOI: https://doi.org/10.12957/rhupe.2016.22840.

8. Nunes BP, Soares MU, Wachs LS, Volz PM, Saes MO, Duro SMS, et al. Hospitalization in older adults: association with multi morbidity, primary health care and private health plan. Rev. Saúde Pública [Internet]. 2017 [cited 2020 May 26]; 51(4):43. DOI: https://doi.org/10.1590/s1518-8787.2017051006646.

9. Baessa CEB, Meireles VC, Balan MAJ. Ocorrência de dermatite associada à incontinência em pacientes internados na unidade de terapia intensiva. Rev. Estima [Internet]. 2014 [cited 2020 Marc 20]; 12(2): Available from: https://www.revistaestima.com.br/index.php/estima/article/view/89.

10. Organização Mundial de Saúde. Classificação internacional de doenças e problemas relacionados à saúde. 10 ed. São Paulo: EDUSP; 2017.

11. World Health Organization. Obesity preventing and managing the global epidemic. Report of a WHO consultation on obesity [Internet]. Geneve; 2000 [cited 2020 May 25]. Available from: http://www.who.int/nutrition/publications/obesity/WHO_TRS_894/en/. 
12. Gray M, Beeckman D, Bliss DZ, Fader M, Logan S, Junkin J, et al. Incontinence-associated dermatitis: a comprehensive review and update. J. Wound Ostomy Continence Nurs. [Internet]. 2012 [cited 2020 Feb 25]; 39(1):61-74. DOI: https://doi.org/10.1097/won.0b013e31823fe246.

13. Wang $X$, Zhang $Y$, Zhang $X$, Zhao $X$, Xian $\mathrm{H}$. Incidence and risk factors of incontinence associated dermatitis among patients in the intensive care unit. J. Clin. Nurs. [Internet]. 2018 [cited 2020 May 13]; 27:4150-7. DOI: https://doi.org/10.1111/jocn.14594.

14. Kottner J, Blume-Peytavi U, Lohrmann C, Halfens R. Associations between individual characteristics and incontinence-associated dermatitis: A secondary data analysis of a multi-centre prevalence study. Int. J. Nurs. Stud. [Internet]. 2014 [cited 2020 May 19]; 51(10):1373-0. DOI: https://doi.org/10.1016/j.ijnurstu.2014.02.012.

15. 15 Newman TB, Browner WS, Hulley SB. Fortalecendo a inferência causal em estudos observacionais. In: Hulley SB, Cummings SR, Browner WS, Grady DG, Newman TB, organizadores. Delineando a pesquisa clínica. 4 ${ }^{\text {a }}$ ed. Porto Alegre (RS): Artmed; 2015. p.124-145.

16. Van Damme N, Clays E, Verhaeghe S, Hecke AV, Beeckman D. Independent Risk Factors for the Development of IncontinenceAssociated Dermatitis (Category 2) in Critically III Patients With Fecal Incontinence: A Cross-Sectional Observational Study in 48 ICU Units. Int. J. Nurs. Stud. [Internet] 2018 [cited 2020 May 19]; 81:30-9. DOI: https://doi.org/10.1016/j.jjnurstu.2018.01.014.

17. Ministério da Saúde $(\mathrm{Br})$. Medidas de prevenção de infecção relacionada à Assistência à Saúde. Ministério da Saúde. Brasília (DF): ANVISA. [Internet]. 2017 [cited 2020 Feb 13]. Available from: http://portal.anvisa.gov.br/documents/33852/3507912/Caderno+4++Medidas+de+Preven\%C3\%A7\%C3\%A30+de+Infec\%C3\%A7\%C3\%A3o+Relacionada+\%C3\%A0+Assist\%C3\%AAncia+\%C3\%A0+Sa\% C3\%BAde/a3f23dfb-2c54-4e64-881c-fccf9220c373.

18. Campbell JL, Coyer FM, Osborne SR. Incontinence-associated dermatitis: a cross-sectional prevalence study in the Australian acute care hospital setting. Int. Wound J. [Internet]. 2016 [cited 2020 May 26]; 13(3):403-1. Available from: https://www.ncbi.nlm.nih.gov/pubmed/24974872.

19. Beeckman D, Van Damme N, Schoonhoven L, Van Lancker A, Kottner J, Beele H, et al. Interventions for preventing and treating incontinence-associated dermatitis in adults. Cochrane Database of Syst. Rev. [Internet]. 2016 [cited 2020 May 15]; 11(11):cd0011627. DOI: https://dx.doi.org/10.1002\%2F14651858.CD011627.pub2.

20. Rosa NM, Inoue KC, Silvino MCS, Olveira MLF. Treatment for dermatitis associated with incontinence in institutionalized elderly: integrative review. Rev. REME [Internet]. 2013 [cited 2020 May 25]; 14(5):1031-40. Available from: http://www.periodicos.ufc.br/rene/article/view/3646/2888.

21. Saurusaitis AD. Análise de custo-minimização do cuidado com a pele na dermatite associada à incontinência. [master thesis]. Rio de Janeiro: Universidade Federal do Estado do Rio de Janeiro; 2017

22. Sokem JAS, Bergamaschi FPR, Watanabe EAMT. Techint about incontinence-associated dermatitis informed by problematization methodology. Rev. Enferm. UERJ [Internet]. 2019 [cited 2020 May 18]; 27:e43727. DOI: https://doi.org/10.12957/reuerj.2019.43727.

23. Beeckman D, Schoonhoven L, Verhaeghe S, Heyneman A, Defloor T. et al. Prevention and treatment of incontinence-associated dermatitis: literature review. J. Adv. Nurs. [Internet]. 2009 [cited 2020 Feb 13]; 65(6):1141-4. DOI: http://dx.doi.org/10.1111/j.1365-2648.2009.04986.x. 